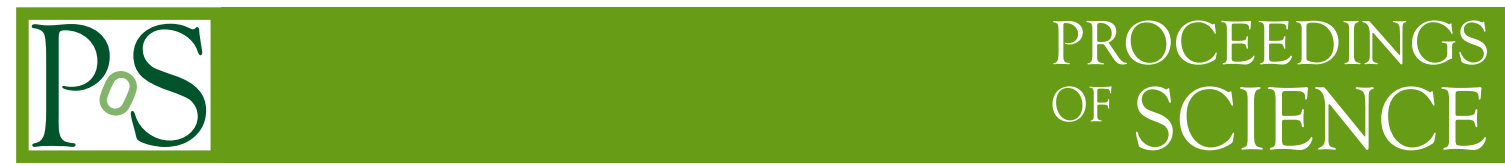

\title{
Strangeness in the nucleon from a mixed action calculation
}

\section{Michael Engelhardt*}

Department of Physics, New Mexico State University, Las Cruces, NM 88003, USA

E-mail: engel@ nmsu.edu

The contributions of strange quarks to the nucleon mass and the nucleon spin are investigated in a mixed action scheme employing domain wall valence quarks and quark loops on MILC asqtad dynamical fermion ensembles. Results are presented for pion masses $495 \mathrm{MeV}$ and $356 \mathrm{MeV}$.

The XXVIII International Symposium on Lattice Field Theory

June 14-19,2010

Villasimius, Sardinia, Italy

${ }^{*}$ Speaker. 


\section{Introduction}

Strange quarks provide a special perspective on nucleon structure due to their absence from the valence content of the nucleon. As a result of this absence, effects of quark-antiquark pair creation and annihilation can be studied in isolation. Correspondingly, considerable efforts to probe strange quarks in the nucleon have been, and continue to be made on the experimental side. On the one hand, electron scattering experiments have provided data on the strange electric and magnetic form factors [1], as well as given some indication of the strange axial structure; the latter, on the other hand, can also be studied in more detail in neutrino scattering experiments, with a combined analysis having been presented in [2]. Considerably enhanced data are expected to emerge from the upcoming neutrino experiments MicroBooNE and MINER $v$ A.

On the side of lattice QCD, calculating strange contributions to nucleon structure counts among the relatively hard problems, since these contributions are determined purely by disconnected diagrams, requiring propagator traces, cf. Fig. 1. On the other hand, among this hard class of problems, strange matrix elements are still the easiest to access, since the quark being propagated in the disconnected loop is heavy, and the associated propagator calculation is therefore less expensive than for light quark loops. Thus, besides the physical relevance of such calculations, they can also serve as initial test cases for exploring techniques to evaluate disconnected contributions to hadron physics more generally. A number of such investigations are being pursued by a variety of groups [5-11]. The effort presented here focuses on the two most fundamental contributions of strange quarks to the properties of the nucleon, namely, their contributions to the nucleon mass and the nucleon spin. The calculational scheme employed is one which has been developed and optimized by the LHP Collaboration [12]. Domain-wall quark propagators are evaluated on HYP-smeared MILC asqtad dynamical quark ensembles. While the use of domain wall fermions implies considerable computational expense, it is expected to yield advantages in terms of mild renormalization and chiral behavior.

\section{Strange matrix elements}

The strange contributions to nucleon mass and spin can be characterized by the matrix elements

$$
f_{T_{s}}=\frac{m_{s}}{m_{N}}\langle N|\bar{s} s| N\rangle \quad \text { and } \quad \Delta s=\left\langle N, i\left|\bar{s} \gamma_{i} \gamma_{5} s\right| N, i\right\rangle
$$

respectively, where $|N, i\rangle$ denotes a nucleon state with spin polarized in the $i$ direction. These matrix elements are obtained from corresponding lattice correlator ratios,

$$
R\left[\Gamma^{n u c}, \Gamma^{o b s}\right](\tau, T)=\frac{\left\langle\left[\Gamma_{\alpha \beta}^{n u c} \Sigma_{\vec{x}} N_{\beta}(\vec{x}, T) \bar{N}_{\alpha}(0,0)\right] \cdot\left[-\Gamma_{\gamma \rho}^{o b s} \Sigma_{\vec{y}} s_{\rho}(\vec{y}, \tau) \bar{s}_{\gamma}(\vec{y}, \tau)\right]\right\rangle}{\left\langle\Gamma_{\alpha \beta}^{\text {unpol }} \Sigma_{\vec{x}} N_{\beta}(\vec{x}, T) \bar{N}_{\alpha}(0,0)\right\rangle}
$$

where $\bar{N}, N$ denote (smeared) nucleon sources and sinks, the sums over spatial position $\vec{x}$ project onto zero momentum nucleon states, the standard minus sign accompanying the quark loop has already become explicit through the reordering of the strange quark fields, and the $\Gamma$ matrices implement nucleon polarization and operator insertion structure. Specifically, the unpolarized nucleon 


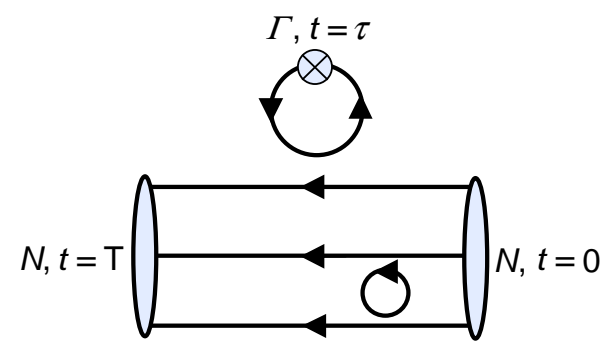

Figure 1: Disconnected contribution to nucleon matrix elements. The nucleon propagates between a source at $t=0$ and a sink at $t=T$; the insertion of $\Gamma \equiv \Gamma^{o b s}$ occurs at an intermediate time $t=\tau$.

two-point function in the denominator of (2.2) is achieved using $\Gamma^{\text {unpol }}=\left(1+\gamma_{4}\right) / 2$; on the other hand, the three-point function in the numerator results from evaluating the correlation between a nucleon propagator and a strange quark loop, as also displayed diagrammatically in Fig. 1. To obtain $f_{T_{s}}$, one calculates

$$
\frac{m_{s}}{m_{N}}\left(R\left[\Gamma^{\text {unpol }}, 1\right](\tau, T)-[\mathrm{VEV}]\right) \equiv R\left\{f_{T_{s}}\right\} \longrightarrow f_{T_{s}}
$$

in the limit $T \gg \tau \gg 0$, where, as indicated, the vacuum expectation value of the quark loop, $[V E V]=\left\langle-\Sigma_{\vec{y}} s_{\gamma}(\vec{y}, \tau) \bar{s}_{\gamma}(\vec{y}, \tau)\right\rangle$ is subtracted; the matrix elements (2.1) are meant to characterize the strange content of the nucleon relative to the vacuum, which itself contains a strange scalar condensate. The nucleon mass $m_{N}$ can be extracted from the nucleon two-point function as a byproduct of the calculation. On the other hand, to obtain $\Delta s$, one calculates

$$
-i \cdot 2 \cdot R\left[\left(-i \gamma_{i} \gamma_{5} / 2\right) \Gamma^{\text {unpol }}, \gamma_{i} \gamma_{5}\right](\tau, T) \equiv R\{\Delta s\} \longrightarrow \Delta s
$$

in the limit $T \gg \tau \gg 0$. In this case, the corresponding vacuum expectation value vanishes, but it can nevertheless be calculationally advantageous to subtract this numerical zero in order to reduce statistical fluctuations. Note that, in (2.4), an average has already been taken over the expectation values obtained using nucleons polarized in the positive and the negative $i$-directions, respectively; averaging the corresponding projectors $\left(1 \mp i \gamma_{i} \gamma_{5}\right) / 2$ (with a relative minus sign) leads to the first argument of $R$ in (2.4). In the numerical calculation, also the polarization axis $i$ will be averaged over the three spatial directions in order to further improve statistics, cf. the description further below. The prefactor 2 in (2.4) is a normalization factor compensating for the fact that the unpolarized nucleon two-point function is always used in the denominator of the ratio (2.2), even when polarized nucleon states are used in the numerator. Finally, the prefactor $(-i)$ incorporates the Wick rotation back to Minkowski space; it compensates for the additional factor $i$ arising in the $\gamma_{5}$ matrix contained in the second argument of $R$ in (2.4) when casting the calculation on the Euclidean lattice.

\section{Lattice setup}

Numerical work was carried out on two $2+1$-flavor asqtad dynamical quark ensembles provided by the MILC collaboration, corresponding to the pion masses $m_{\pi}=356 \mathrm{MeV}$ and $m_{\pi}=$ 


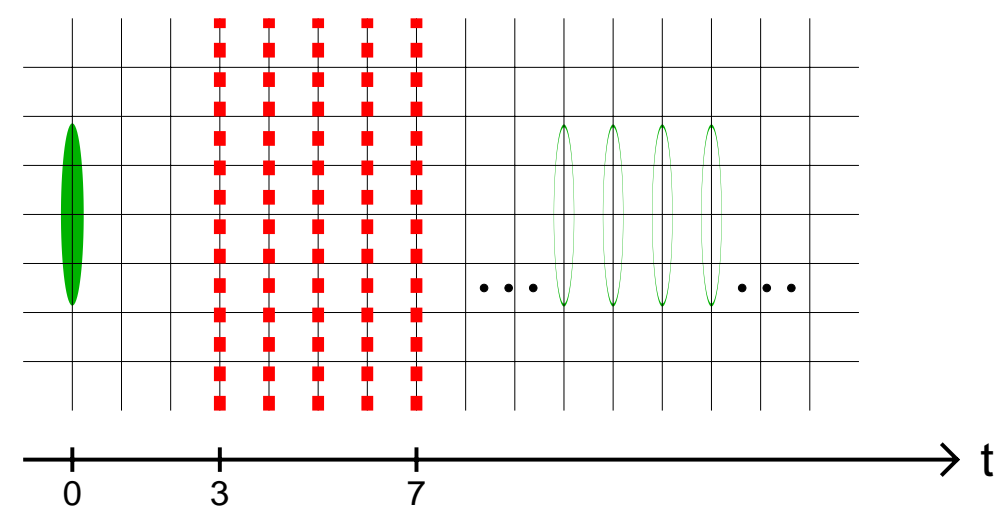

Figure 2: Setup of the lattice calculation. The nucleon source is located at lattice time $t=0$. The operator insertion time $\tau$ is averaged over $t=3, \ldots, 7$; accordingly, stochastic sources are distributed over the bulk of the lattice in this entire time range. The temporal position $T$ of the nucleon sink is variable.

$495 \mathrm{MeV}$. These ensembles contained 448 and $48620^{3} \times 64$ lattices, respectively, with lattice spacing $a=0.124 \mathrm{fm}$. The configurations were HYP-smeared for the purpose of this calculation. The nucleon two-point functions and strange quark loops were evaluated using domain wall quarks. The lattice setup employed is depicted in Fig. 2. To enhance statistics, the operator insertion time $\tau$ in (2.2) was averaged over five time slices, $t=3, \ldots, 7$ (where $t=0$ corresponds to the nucleon source position). To implement this average, complex $Z(2)$ stochastic sources, introduced to the evaluate the strange quark loop propagator trace, were distributed over the bulk of the lattice within this entire temporal range. For $m_{\pi}=356 \mathrm{MeV}, 1200$ stochastic sources per configuration were used, for $m_{\pi}=495 \mathrm{MeV}, 600$ stochastic sources $^{1}$. This rigid temporal setup, chosen to allow for maximal statistics, does not permit a variation of the operator insertion time in order to test for a plateau; it is motivated by previous extensive experience with connected diagrams in the same scheme [12], which suggests that the nucleon ground state has been filtered out at $t=3$ to a sufficient degree as to render the associated systematic uncertainty small compared to the statistical uncertainty of the present calculation. On the other hand, a residual opportunity to test the dependence of the results specifically on the operator-sink separation is given; below, results for the relevant correlator ratios will be shown as a function of variable sink position $T$, with the expectation that the asymptotic behavior will be reached for sink positions $T \geq 10$.

Besides statistical uncertainties stemming from the stochastic evaluation of the quark loop, the observables studied here exhibit substantial gauge fluctuations. To accumulate statistics sufficient to overcome these fluctuations, it is necessary to evaluate multiple samples of the correlator ratios of interest per given gauge configuration. Thus, for given source time slice, not one, but several (eight in the case of $m_{\pi}=356 \mathrm{MeV}$, four in the case of $m_{\pi}=495 \mathrm{MeV}$ ) different samples were obtained by varying the spatial source position. Furthermore, since the scheme depicted in Fig. 2 requires a much smaller temporal extent than available on the lattice employed, the entire scheme was replicated three times on separate temporal regions of the lattice. Altogether, therefore, 24 correlator ratio samples were obtained per lattice gauge configuration in the case of $m_{\pi}=356 \mathrm{MeV}$,

\footnotetext{
${ }^{1}$ These high statistics in the stochastic sources mainly serve to improve the signal for $\Delta s$; the stochastic estimator for the scalar matrix element, by contrast, converges rapidly and the statistical uncertainty in $f_{T_{s}}$ is dominated by gauge fluctuations.
} 

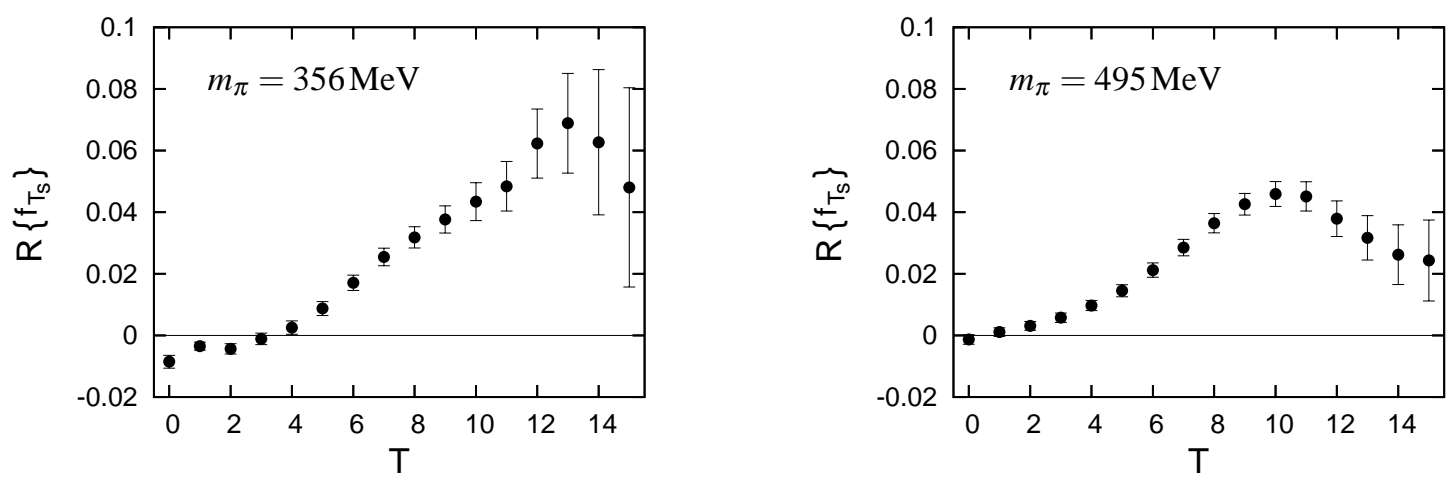

Figure 3: Correlator ratio $R\left\{f_{T_{S}}\right\}$ as a function of sink time $T$, for the two pion masses considered.
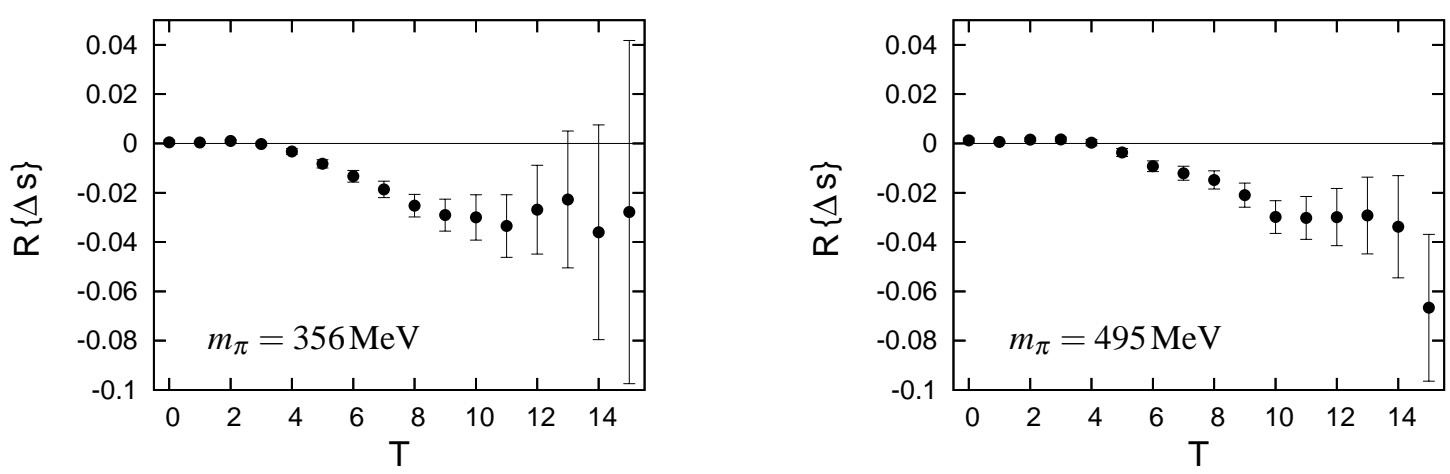

Figure 4: Correlator ratio $R\{\Delta s\}$ as a function of $\operatorname{sink}$ time $T$, for the two pion masses considered.

and 12 in the case of $m_{\pi}=495 \mathrm{MeV}$. Finally, as already indicated in the previous section, $\Delta s$ was averaged over three separate polarization axes $i$, corresponding to the coordinate axes.

\section{Numerical results and conclusions}

Figs. 3 and 4 display, as a function of sink time $T$, the correlator ratios $R\left\{f_{T_{s}}\right\}$ and $R\{\Delta s\}$, cf. (2.3) and (2.4), averaged in the fashion described in the preceding section; for large $T$, these quantities yield the strange contributions to the nucleon mass and spin, $f_{T_{s}}$ and $\Delta s$. Before reaching the physically most relevant region $T \geq \tau$ (where $\tau$ denotes the temporal location(s) of the operator insertion), the ratios start out at vanishing values near $T=0$, then gather up magnitude as the source-sink time interval includes an increasing portion of the stochastic source region contributing to the quark loop. In the region $T \geq \tau$, the correlator ratios are expected to level off to approach their asymptotic value for large sink times. On the the other hand, in this region, statistical fluctuations become appreciable, and there is therefore only a short time window in which one can hope to observe this behavior.

In the case of $\Delta s$, cf. Fig. 4, this expectation is confirmed rather well. Quantitatively, the estimates of $\Delta s$ obtained either from the correlator ratio at sink time $T=10$ or from the average over the correlator ratios at sink times $T=10, \ldots, 14$ yield almost identical results,
At $m_{\pi}=356 \mathrm{MeV}$ :
$\left.\Delta s\right|_{T=10}=-0.030(9)$
$\left.\Delta s\right|_{T=10 \ldots 14}=-0.030(19)$
At $m_{\pi}=495 \mathrm{MeV}$ :
$\left.\Delta s\right|_{T=10}=-0.030(7)$
$\left.\Delta s\right|_{T=10 \ldots 14}=-0.031(11)$ 

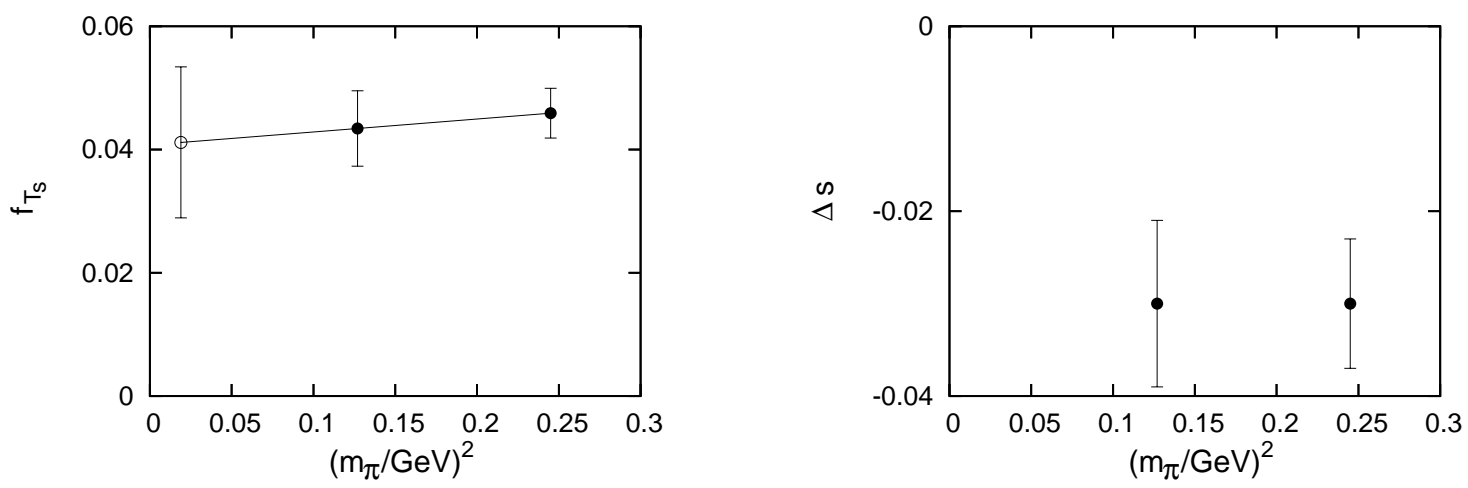

Figure 5: Pion mass dependence of the results for $f_{T_{s}}$ and $\Delta s$. Filled circles represent measured data; open circle in left-hand panel shows chiral extrapolation of the data to the physical pion mass, cf. main text.

where the error estimate in the sink-time averaged case is the jackknife error extracted by performing the sink time average configuration by configuration, i.e., the fact that correlator ratios at different sink times are not independent is taken into account.

On the other hand, in the case of $f_{T_{s}}$, the behavior of the correlator ratios as a function of sink time is not as clear-cut. At $m_{\pi}=356 \mathrm{MeV}$, the correlator ratio for $T>10$ considerably overshoots the value at $T=10$, whereas at $m_{\pi}=495 \mathrm{MeV}$, the correlator ratio for $T>10$ decreases again compared to its $T=10$ value. Quantitatively, the comparison analogous to the one performed for $\Delta s$ above yields

$$
\begin{array}{lll}
\text { At } m_{\pi}=356 \mathrm{MeV}: & \left.f_{T_{s}}\right|_{T=10}=0.043(6) & \left.f_{T_{s}}\right|_{T=10 \ldots 14}=0.057(11) \\
\text { At } m_{\pi}=495 \mathrm{MeV}: & \left.f_{T_{s}}\right|_{T=10}=0.046(4) & \left.f_{T_{s}}\right|_{T=10 \ldots 14}=0.037(5)
\end{array}
$$

The fact that the deviations from the expected plateau behavior occur in opposite directions for the two pion masses may be an indication that they are caused by statistical fluctuations; also the error estimates in (4.3),(4.4) are still compatible with this possibility. In view of the fact that correlator ratios at different sink times are not independent of each other, the rather smooth behavior of the correlator ratio in the case of $m_{\pi}=495 \mathrm{MeV}$ does not necessarily contradict an explanation in terms of statistical fluctuations. Currently, a doubling of the statistics for the $m_{\pi}=495 \mathrm{MeV}$ case is being pursued to further explore this issue.

Fig. 5 summarizes the results obtained as a function of pion mass, using the correlator ratios at sink time $T=10$ to estimate the observables; for the case of $f_{T_{s}}$, the above discussion of the uncertainties involved in identifying an asymptotic value of the corresponding correlator ratio should be kept in mind. For $f_{T_{s}}$, also a tentative extrapolation to the physical pion mass is displayed; since the strange scalar matrix element in the nucleon is related via the Feynman-Hellman theorem to the derivative of the nucleon mass with respect to the strange quark mass, the chiral behavior follows from differentiation of nucleon mass formulae obtained in chiral effective theory [7]. The leading dependence is linear in the light quark mass, i.e., in $m_{\pi}^{2}$; this was used in the extrapolation shown in the left-hand panel of Fig. 5. The extrapolated value is $f_{T_{s}}=0.041(12)$, corresponding to $m_{s}\langle N|\bar{s} s| N\rangle=39(12) \mathrm{MeV}$.

On the other hand, while no attempt at extrapolating $\Delta s$ in the right-hand panel of Fig. 5 has been made, no significant variation with pion mass is seen. It should be noted that the strange axial 
current entering the calculation of $\Delta s$ requires renormalization, which has not been included in the results presented here; however, for the lattice scheme used in this work, axial current renormalization constants translating to the $\overline{M S}$ scheme at a scale of $2 \mathrm{GeV}$ are consistently very close to 1.1 , over a wide range of quark masses [12]. It is therefore expected that the results for $\Delta s$ only acquire a mild $10 \%$ enhancement when translated to the standard $\overline{M S}$ scheme. Thus, no evidence for unnaturally large strange quark contributions to nucleon spin is seen in the present calculation.

Of course, it should be noted that no attempt has been made at this point to quantify several other sources of systematic uncertainty, such as the dependence on the lattice spacing, lattice size, and, in particular, the fact that the strange quark mass in the gauge ensembles used lies appreciably above the physical strange quark mass. Data on the strange quark mass dependence reported in [7], translated to the present scheme, indicate that matrix elements such as $\langle N|\bar{s} s| N\rangle$ acquire a correction amounting to about $15 \%$ (which in $f_{T_{s}}$ is (over)compensated by the $m_{s}$ prefactor); it thus appears reasonable to conjecture a $15 \%$ systematic error also for $\Delta s$ from this source, about half the magnitude of the statistical uncertainty.

\section{Acknowledgments}

The computations required for this investigation were carried out at the Encanto computing facility operated by NMCAC, using the Chroma software suite [13]. This work was supported by the U.S. DOE under grant DE-FG02-96ER40965.

\section{References}

[1] J. Liu, R. D. McKeown and M. Ramsey-Musolf, Phys. Rev. C 76 (2007) 025202.

[2] S. Pate, D. McKee and V. Papavassiliou, Phys. Rev. C 78 (2008) 015207.

[3] A. Airapetian et al. (HERMES collaboration), Phys. Rev. D 75 (2007) 012007.

[4] A. Airapetian et al. (HERMES collaboration), Phys. Lett. B666 (2008) 446.

[5] G. Bali, S. Collins and A. Schäfer, PoS LAT2009 (2009) 149.

[6] G. Bali, S. Collins and A. Schäfer, Comput. Phys. Commun. 181 (2010) 1570.

[7] D. Toussaint and W. Freeman, Phys. Rev. Lett. 103 (2009) 122002.

[8] T. Doi, M. Deka, S.-J. Dong, T. Draper, K.-F. Liu, D. Mankame, N. Mathur and T. Streuer, Phys. Rev. D 80 (2009) 094503.

[9] R. Babich, R. Brower, M. Clark, G. Fleming, J. Osborn and C. Rebbi, PoS LATTICE2008 (2008) 160.

[10] K. Takeda, S. Aoki, S. Hashimoto, T. Kaneko, T. Onogi and N. Yamada, PoS LAT2009 (2009) 141.

[11] H. Ohki, S. Aoki, H. Fukaya, S. Hashimoto, T. Kaneko, H. Matsufuru, J. Noaki, T. Onogi, E. Shintani and N. Yamada, PoS LAT2009 (2009) 124.

[12] J. D. Bratt, R. G. Edwards, M. Engelhardt, P. Hägler, H.-W. Lin, M.-F. Lin, H. B. Meyer, B. Musch, J. W. Negele, K. Orginos, A. V. Pochinsky, M. Procura, D. G. Richards, W. Schroers and S. N. Syritsyn, Phys. Rev. D 82 (2010) 094502.

[13] R. G. Edwards and B. Joó (LHPC and UKQCD Collaborations), Nucl. Phys. Proc. Suppl. 140 (2005) 832. 